\title{
Hemodynamic changes after transcatheter aortic valve implantation during sequential follow-ups in patients with bicuspid aortic valve compared with tricuspid aortic valve
}

\author{
Tian-Yuan Xiong, Ming-Xia Zheng, Xin Wei, Yi-Jian Li, Yan-Biao Liao, \\ Zhen-Gang Zhao, Yuan-Ning Xu, Hong Tang, Yuan Feng, Mao Chen \\ Department of Cardiology, West China Hospital, Sichuan University, China
}

\begin{abstract}
Background: To investigate the individual sequential hemodynamic changes after transcatheter aortic valve implantation (TAVI), especially for patients with bicuspid aortic valve (BAV), in comparison with tricuspid aortic valve (TAV).

Methods: The study population comprised 85 patients with severe aortic stenosis who underwent TAVI for BAV $(n=49)$ or TAV $(n=36)$ with at least two serial echocardiographic follow-ups. Doppler echocardiography was scheduled to be performed at discharge and 1, 3, 6 months and 1 year after the procedure. $\Delta$ peak transvalvular velocities and $\Delta$ mean transvalvular gradients were calculated as the difference at follow-up time points and discharge. Paravalvular leak (PVL) was assessed as another indicator for prosthesis performance.

Results: Comparisons between patients with BAV and TAV revealed similar gradient performances $(1.00$ [-2.00, 2.00] vs. $1.00[-0.25,5.00] \mathrm{mm} \mathrm{Hg}, p=0.57$ at 1 month; $-0.71 \pm 7.52$ vs. $1.55 \pm 3.97$ $\mathrm{mm} \mathrm{Hg}, p=0.21$ at 3 months; $0.96 \pm 7.81$ vs. $1.53 \pm 5.85 \mathrm{~mm} \mathrm{Hg}, p=0.79$ at 6 months; 1.00 [-0.50, $2.25]$ vs. 3.00 [-0.50, 7.50] $\mathrm{mm} \mathrm{Hg}, p=0.07$ at 1 year). Moreover, the incidence of $\geq$ mild PVL was not significantly different in patients with BAV and TAV during follow-up (34.88\% vs. $19.35 \%, p=0.14$ at 1 month; $45.83 \%$ vs. $27.27 \%, p=0.19$ at 3 months; $30.00 \%$ vs. $23.53 \%, p=0.89$ at 6 months; $30.00 \%$ vs. $17.65 \%, p=0.56$ at 1 year).
\end{abstract}

Conclusions: TAVI is effective and applicable in BAV anatomy with sustained and acceptable mid-term prosthesis hemodynamic performance. (Cardiol J 2017; 24, 4: 350-357)

Key words: aortic stenosis, transcatheter aortic valve implantation, hemodynamics

\section{Introduction}

During the past decade, transcatheter aortic valve implantation (TAVI) has evolved rapidly as an alternative treatment option to conventional aortic valve replacement (AVR) for those who are deemed inoperable or at high risk for surgery. The safety and effectiveness of this new technique has been demonstrated, as well as sustained transvalvular hemodynamics post-TAVI has also shown signifi- cant improvement in symptoms [1,2]. Despite data favoring low transprosthetic gradients and large prosthetic valve effective orifice area in several studies [3-6], the assumption that implantation of a percutaneous bioprosthesis within the native valve might lead to incomplete expansion of the prosthetic valve [7] is still worrisome, since the evidence in individual continuous hemodynamic change has been lacking. This is especially true in bicuspid aortic valve (BAV) patients for their

Address for correspondence: Mao Chen, MD, PhD, Department of Cardiology, West China Hospital, Sichuan University, 37 Guoxue Alley, Chengdu, 610041, PR China, tel: 86-28-85423362, fax: 86-28-85423170, e-mail: hmaochen@vip.sina.com Received: 29.09.2016 Accepted: 29.12.2016 
pre-existing abnormal cusp fusion, heavily calcified leaflets and possible raphe, all of which, could theoretically jeopardize the prosthetic valve function [8].

Transvalvular gradients on Doppler echocardiography has been shown to correlate well with invasive measures for assessing valve function and hemodynamics after AVR [9]. In this study, we sought to use mid-term serial echocardiographic follow-up data to identify the changes of these two hemodynamic parameters and to compare the differences between BAV patients and their tricuspid counterparts.

\section{Methods}

Data from patients with severe symptomatic aortic stenosis who underwent TAVI in our center with at least two serial echocardiographic followups were retrospectively collected into two groups according to valve morphology. BAV patients were not denied for TAVI at our center. The definition and classifications of BAV have been described elsewhere [8]. It is characterized as the abnormal aortic valve morphology consisting of two functional cusps with less than three zones of parallel apposition between cusps. The number and spatial orientation of the raphe were used to classify this morphology. Possible BAV patients were identified on echocardiography, but measurements of the aortic annulus, diagnosis and classification of BAV were confirmed by interpreting pre-procedural multi-slice computed tomography (MSCT) with commercially available software, OsiriX (OsiriX Foundation, Geneva, Switzerland), by methods proposed earlier [10]. All patients were evaluated by a multidisciplinary team beforehand. If the diagnosis of severe aortic stenosis was confirmed on echocardiography and considered by cardiac surgeons to be inoperable or at high surgical risk, TAVI was considered. The study protocol was approved by the institutional review board and all patients provided written, signed consent.

MSCT was routinely performed to evaluate the anatomical suitability for TAVI and guide the selection of access route and prosthesis size. TAVI was performed with self-expanding systems, the first-generation CoreValve (Medtronic, Minneapolis, Minnesota) or the Venus A-Valve (Venus MedTech Inc., Hangzhou, China). The decision for using one of the two valves depended on valve availability. The Venus A-Valve is a self-expanding nitinol stent frame carrying a trileaflet porcine bioprosthetic valve. The delivery system is $18-\mathrm{Fr}$ and can be delivered sheathless by the transfemoral as well as transaxillary/transsubclavian approach, and with a sheath for the transaortic approach. The radial force of expansion for the inflow was increased, enabling a more consistent device expansion in the presence of extreme aortic valve calcification [11]. All procedures were performed in the hybrid operating room with patients under general anesthesia. Intraprocedural transesophageal echocardiography (TEE) was performed to assess the performance of the prosthetic valve in the absence of contraindication. The transfemoral approach was the defaulted access, and if it was not feasible, the transsubclavian approach was considered as the alternative. Procedural details of TAVI with the self-expanding devices have been described previously $[12,13]$.

Doppler echocardiography was systematically performed at baseline and at hospital discharge in all patients. The timing of echocardiographic exams at follow-up was set at 1 months, 3 months, 6 months and 1 year after procedure and then yearly. Left ventricular ejection fraction (LVEF), stroke volume (SV), transvalvular gradients and peak velocity were measured following the methods previously described [14]. $\Delta$ peak transvalvular velocities were calculated as the difference at the four follow-up intervals and discharge if available. Results were shown as $\Delta \mathrm{V}_{1}$ for peak velocity at discharge subtracted the value at 1 month after procedure, $\Delta \mathrm{V}_{2}$, $\Delta \mathrm{V}_{3}, \Delta \mathrm{V}_{4}$ and so on. Likewise, $\Delta$ mean transvalvular gradient was calculated as the difference at the four follow-up intervals and discharge if available. Results were shown as $\Delta \mathrm{G}_{1}$ for mean transvalvular gradient at discharge subtracted the value at 1 month after procedure, $\Delta \mathrm{G}_{2}, \Delta \mathrm{G}_{3}, \Delta \mathrm{G}_{4}$ and so on. Prosthetic valve function and paravalvular leak (PVL) were also assessed as an indicator for prosthesis performance in accordance to the updated Valve Academic Research Consortium (VARC) criteria [15] and shown as none (0), trace (1) and mild or more than mild $(\geq 2)$ in this study.

\section{Statistical analysis}

Continuous variables are reported as mean \pm standard deviation or median (interquartile range). Categorical variables were presented as frequencies and percentages. Differences in proportions were assessed using the $\chi^{2}$ test or Fisher's exact test. To test for differences in means between groups with continuous variables, an unpaired Student's t test (one-way ANOVA was used if the comparison was among more than two groups) or Mann-Whitney or Kruskal-Wallis-H 
non-parametric test was used. To test if two variables vary together, simple scatter plot was used to see whether potential correlation existed, and if so, Pearson correlation calculations or Spearman nonparametric correlation would be utilized for the correlation coefficient. All computations relied on commercially available software (SPSS IBMS v. 21 for Mac; SPPS Inc., Chicago, IL, USA), with statistical significance set at two-tailed 0.05.

\section{Results}

A total of 85 patients were identified. The mean age was 74.00 years and $50(58.82 \%)$ patients were male. A total of $57.65 \%(49 / 85)$ of patients were with BAV (30 with type $0 ; 19$ with type 1 including 14 left-right, 4 right-non and 1 left-non), which was in line with a previously reported cohort [16]. The mean Society of Thoracic Surgeons Predicted Risk of Mortality (STS-PROM) score was $7.38 \pm 0.42 \%$. Nearly all patients $(97.65 \%)$ underwent TAVI with the transfemoral approach. A total of $47.06 \%(40 / 85)$ of patients received the Medtronic CoreValve, while the others received the Venus A-valve. The demographic and procedural characteristics of the study population are summarized in different valve morphologies in Table 1 . At the time of data retrieval, there were 80 patients with transthoracic echocardiography (TTE) at discharge. The numbers of available TTE follow-ups were 74, 46, 47 and 47 at 1 month, 3 months, 6 months and 1 year after the procedure, respectively.

$\Delta \mathrm{V}_{1}=0.005(-0.138,0.200) \mathrm{m} / \mathrm{s}, \Delta \mathrm{V}_{2}=0.000$ $(-0.190,0.290) \mathrm{m} / \mathrm{s}, \Delta \mathrm{V}_{3}=0.055(-0.300,0.338) \mathrm{m} / \mathrm{s}$, $\Delta \mathrm{V}_{4}=0.200(-0.100,0.340) \mathrm{m} / \mathrm{s}$ with no significant differences among them $(\mathrm{p}=0.53)$. At the same follow-up period, no significant differences were detected between BAV and tricuspid aortic valve (TAV) patients for $\Delta \mathrm{V}$ at 1 month $(0.000[-0.185$, $0.198]$ vs. $0.040[-0.090,0.300] \mathrm{m} / \mathrm{s}, \mathrm{p}=0.35$; at 3 months $(0.000[-0.308,0.300]$ vs. $0.000[-0.130$, $0.235] \mathrm{m} / \mathrm{s}, \mathrm{p}=0.75)$; and at 6 months $(0.100$ $[-0.300,0.400]$ vs. $-0.020[-0.400,0.313] \mathrm{m} / \mathrm{s}$, $\mathrm{p}=0.67)$, except for 1 -year follow-up $(0.03[-0.180$, $0.300]$ vs. $0.275[0.070,0.530] \mathrm{m} / \mathrm{s}, \mathrm{p}=0.04)$. On the other hand, $\Delta \mathrm{G}_{1}=1.00(-0.50,3.00) \mathrm{mm}$ $\mathrm{Hg}, \Delta \mathrm{G}_{2}=1.00(-2.25,3.00) \mathrm{mm} \mathrm{Hg}, \Delta \mathrm{G}_{3}=1.00$ $(-2.00,5.00) \mathrm{mm} \mathrm{Hg} \Delta \mathrm{G}_{4}=2.00(0.00,3.00) \mathrm{mm} \mathrm{Hg}$ with no significant differences among them $(p=0.79)$. Also, differences between BAV and TAV patients for $\Delta \mathrm{G}$ did not reach statistical significance at all follow-up intervals: at 1 month $(1.00[-2.00$, $2.00]$ vs. $1.00[-0.25,5.00] \mathrm{mm} \mathrm{Hg}, \mathrm{p}=0.57)$; at
3 months, $-0.71 \pm 7.52$ vs. $1.55 \pm 3.97 \mathrm{~mm} \mathrm{Hg}$, $\mathrm{p}=0.21$; at 6 months, $0.96 \pm 7.81$ vs. $1.53 \pm 5.85$ $\mathrm{mm} \mathrm{Hg}, \mathrm{p}=0.79$; and at 1 year $(1.00[-0.50,2.25]$ vs. $3.00[-0.50,7.50] \mathrm{mm} \mathrm{Hg}, \mathrm{p}=0.07)$. These data were illustrated in Figures 1 and 2.

As for PVL, grade $\geq 2$ PVL was recorded in $34.1 \%(29 / 85)$ of patients on TEE after the procedure $(38.8 \%$ in BAV and $27.8 \%$ in TAV, $\mathrm{p}=0.29$ ). This result was achieved with post-dilation and the valve-in-valve procedure. Total of $44.9 \%$ (22/49) BAV patients underwent post-dilation, while it was $41.7 \%(15 / 36)$ in TAV $(p=0.29)$. Two $(5.6 \%)$ TAV patients underwent valve-in-valve procedure, there were $7(14.3 \%)$ patients in BAV $(\mathrm{p}=0.29)$. No differences in the distribution of PVL severity was seen from the time of discharge (patients with grade $\geq 2$ PVL in TAV and BAV groups: $33.33 \%$ vs. $31.82 \%, \mathrm{p}=0.89$ at discharge; $19.35 \%$ vs. $34.88 \%$, $\mathrm{p}=0.14$ at 1 month; $27.27 \%$ vs. $45.83 \%, \mathrm{p}=0.19$ at 3 months; $23.53 \%$ vs. $30.00 \%, p=0.89$ at 6 months; $17.65 \%$ vs. $30.00 \%, \mathrm{p}=0.56$ at 1 year). See Figure 3 for details.

Scatter plots for LVEF or SV and peak velocities in order to detect possible correlations on all four follow-up intervals were drawn (figures not shown) with only low correlations between peak velocity and SV at discharge and 1-year follow-up reaching significance $(r=0.261, p=0.02$ and $\mathrm{r}=0.403, \mathrm{p}<0.01$ respectively).

\section{Discussion}

The main findings of this study are that: 1) Sustained hemodynamic changes are observed as peak velocity and transvalvular gradient differences between discharge and follow-up within 1 year after the procedure; 2) BAV does not seem to alter hemodynamic changes comparing with its TAV counterparts in TAVI with the self-expanding transcatheter heart valve (THV); 3) The incidence of $\geq$ grade $2 \mathrm{PVL}$ was not significantly different in BAV patients during follow-up.

Unlike AVR, some studies suggested TAVI results in immediate and sustained relief in pressure overload and improved left ventricular systolic function, with continued regression of hypertrophy over time [1]. However, most previous studies have only reported the hemodynamic performance at each interval without clearly showing the hemodynamic changes for individual patients between two follow-up intervals. As a continuous process, the interactions between native valve, aortic annulus and surrounding structures, THV and its impact on hemodynamics need to be further delineated. 
Table 1. Clinical characteristics of the study population and procedure data.

\begin{tabular}{|c|c|c|c|}
\hline Parameters & $\operatorname{BAV}(n=49)$ & $\operatorname{TAV}(n=36)$ & $\mathbf{P}$ \\
\hline Age [years] & $72.65 \pm 6.68$ & $73.28 \pm 7.42$ & 0.30 \\
\hline Male & $31(63.3 \%)$ & $19(52.8 \%)$ & 0.33 \\
\hline Weight [kg] & $57.84 \pm 9.84$ & $56.76 \pm 10.26$ & 0.70 \\
\hline Height $[\mathrm{m}]$ & $1.60 \pm 0.09$ & $1.61 \pm 0.10$ & 0.20 \\
\hline STS score [\%] & $6.01(4.39,9.23)$ & $8.28(4.89,9.25)$ & 0.34 \\
\hline NYHA III-IV & $44(89.8 \%)$ & $32(88.9 \%)$ & 0.99 \\
\hline \multicolumn{4}{|l|}{ Antecedents } \\
\hline Myocardial infarction & $2(4.1 \%)$ & $1(2.8 \%)$ & 0.99 \\
\hline Prior PCl & $3(6.1 \%)$ & $2(5.6 \%)$ & 0.99 \\
\hline Atrial fibrillation & $10(20.4 \%)$ & $5(13.9 \%)$ & 0.44 \\
\hline \multicolumn{4}{|l|}{ Comorbidity } \\
\hline Hypertension & $22(44.9 \%)$ & $19(52.8 \%)$ & 0.47 \\
\hline Diabetes & $13(26.5 \%)$ & $9(25.0 \%)$ & 0.87 \\
\hline Chronic lung disease & $28(57.1 \%)$ & $27(75.0 \%)$ & 0.09 \\
\hline Coronary artery disease & $15(30.6 \%)$ & $14(38.9 \%)$ & 0.43 \\
\hline Peripheral vascular disease & $19(38.8 \%)$ & $11(30.6 \%)$ & 0.43 \\
\hline Chronic kidney disease & $6(12.2 \%)$ & $6(16.7 \%)$ & 0.56 \\
\hline \multicolumn{4}{|l|}{ Pre-procedural TEE } \\
\hline \multicolumn{4}{|l|}{ Left ventricular function* } \\
\hline Normal & $33(67.3 \%)$ & $29(80.6 \%)$ & 0.18 \\
\hline Moderate & $14(28.6 \%)$ & $5(13.9 \%)$ & 0.11 \\
\hline Impaired & $2(4.1 \%)$ & $2(5.6 \%)$ & 0.99 \\
\hline \multicolumn{4}{|l|}{ Peak velocity } \\
\hline Pre-procedure [m/s] & $5.10(4.55,5.75)$ & $4.90(4.31,5.20)$ & 0.05 \\
\hline Post-procedure [m/s] & $2.40(2.00,2.80)$ & $2.48(2.12,2.80)$ & 0.92 \\
\hline \multicolumn{4}{|l|}{ Annulus on CT } \\
\hline Maximal diameter $[\mathrm{mm}]$ & $27.53 \pm 3.43$ & $27.13 \pm 3.36$ & 0.59 \\
\hline Minimal diameter [mm] & $21.88 \pm 2.92$ & $20.51 \pm 2.54$ & 0.03 \\
\hline Eccentricity index $[\%]^{\#}$ & $20.15 \pm 8.91$ & $24.18 \pm 6.05$ & 0.02 \\
\hline \multicolumn{4}{|l|}{ Procedure } \\
\hline CoreValve & $25(51.0 \%)$ & $15(41.7 \%)$ & 0.58 \\
\hline \multicolumn{4}{|l|}{ Valve size } \\
\hline $23 \mathrm{~mm}$ & $1(2.1 \%)$ & $1(2.8 \%)$ & 0.99 \\
\hline $26 \mathrm{~mm}$ & $22(44.9 \%)$ & $15(41.7 \%)$ & 0.77 \\
\hline $29 \mathrm{~mm}$ & $15(30.6 \%)$ & $13(36.1 \%)$ & 0.59 \\
\hline $31 / 32 \mathrm{~mm}$ & $11(22.4 \%)$ & $7(19.4 \%)$ & 0.74 \\
\hline
\end{tabular}

* Left ventricular function was determined by visual assessment on transesophageal echocardiography (TEE) and classified as normal in case of an estimated ejection fraction of $>50 \%$, moderate if $30 \%$ to $50 \%$, and impaired if $<30 \%$; ${ }^{*}$ Eccentricity index $=\left(1-D_{\min } / D_{\text {max }}\right) \times 100$; BAV - bicuspid aortic valve; CT — computed tomography; TAV - tricuspid aortic valve; NYHA — New York Heart Association;

$\mathrm{PCl}$ - percutaneous coronary intervention; STS - Society of Thoracic Surgeons

Because of its asymmetric annulus anatomy, BAV is traditionally regarded as a relative contradiction for TAVI for the assumption that this challenging anatomy could exert an increased risk of uneven expansion of THV and may lead to leaflet deformity and PVL. Zegdi et al. [7] described a non-circular stent shape in $85 \%$ of patients with BAV whereas it was $32 \%$ in TAV patients in a study where they put non-commercial self-expanding stent in 16 patients with BAV and 19 patients with TAV before AVR. This proposed elliptical shape was later observed in several studies on 

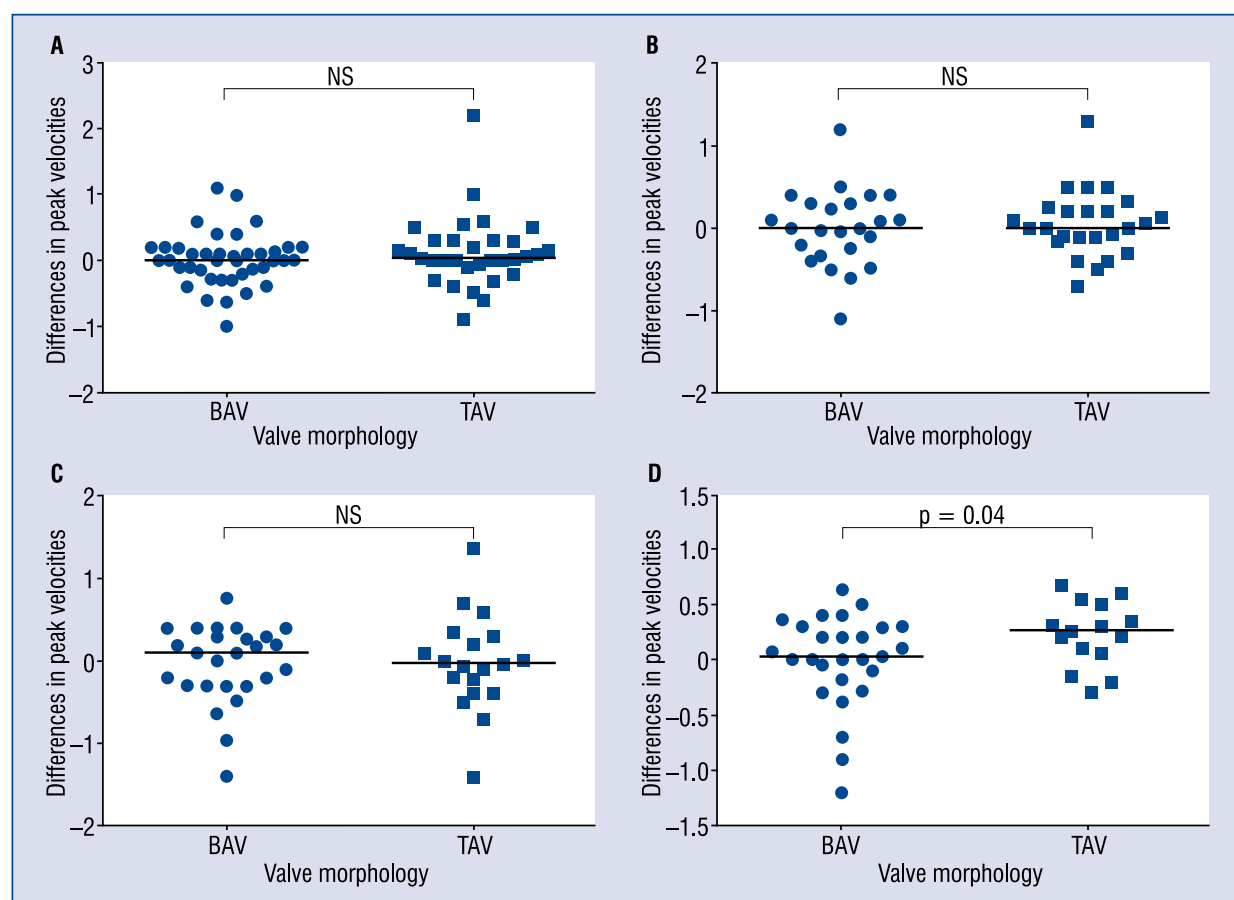

Figure 1. Scatter plots for individual differences in peak velocities at four follow-up time points grouped by valve morphology. Each circle (for bicuspid aortic valve [BAV]) or square (for tricuspid aortic valve [TAV]) in this figure stands for the difference in peak velocity between follow-up at that time and at discharge, intentionally arranged by two morphology groups for readers to appreciate variations from different anatomical features. NS in the figure represents non-significant $p$ value in the student $t$ test or Mann-Whitney test; A. $\Delta \mathrm{V}_{1} ;$ B. $\Delta \mathrm{V}_{2} ;$ C. $\Delta \mathrm{V}_{3} ;$ D. $\Delta \mathrm{V}_{4}$.

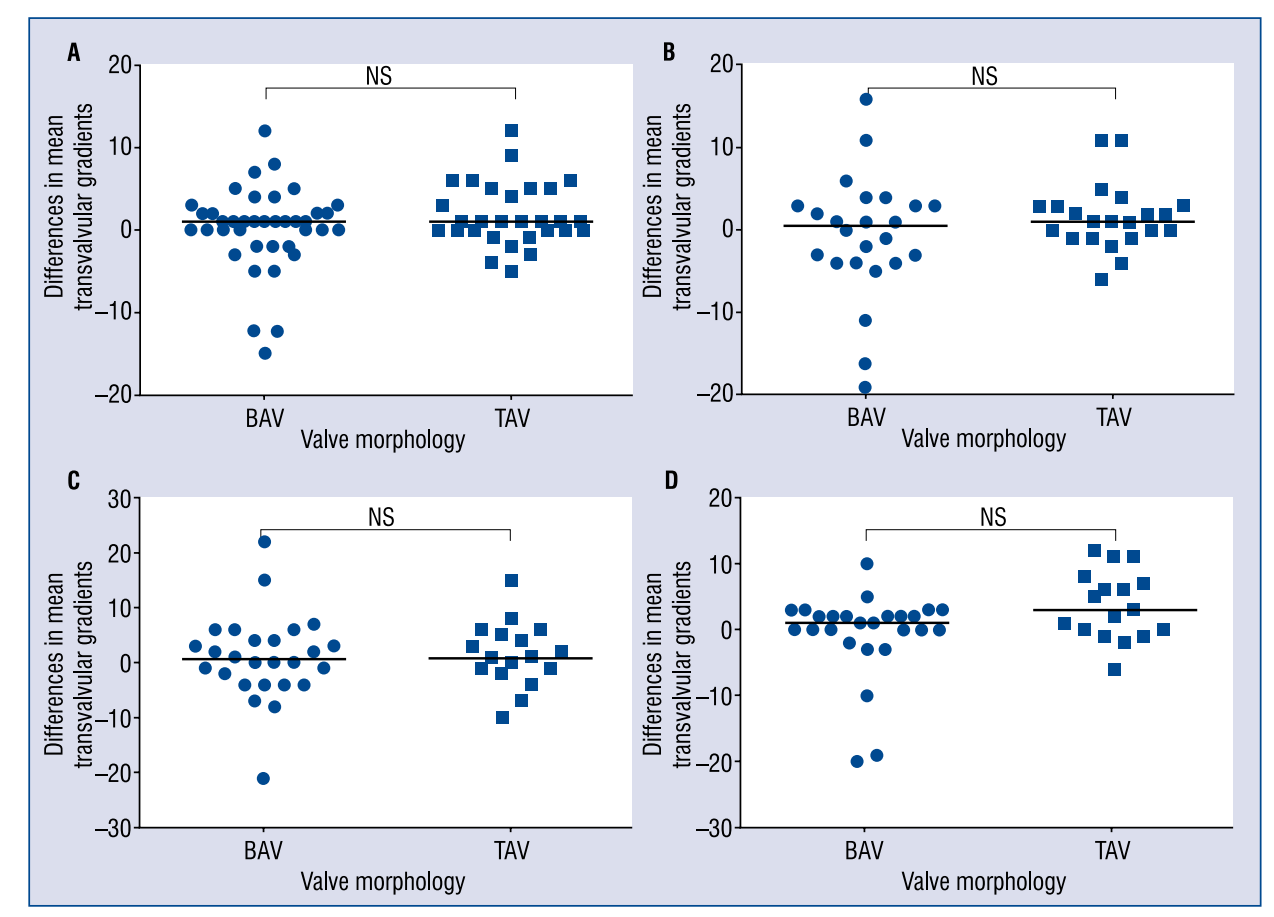

Figure 2. Scatter plots for individual differences in mean transvalvular gradients at four follow-up time points grouped by valve morphology. Each circle (for bicuspid aortic valve [BAV]) or square (for tricuspid aortic valve [TAV]) in this figure stands for the difference in transvalvular gradient between follow-up at that time and at discharge, intentionally arranged by two morphology groups for readers to appreciate variations from different anatomical features. NS in the figure represents non-significant $p$ value in the student $t$ test or Mann-Whitney test; $A . \Delta G_{1} ;$ B. $\Delta G_{2} ;$ C. $\Delta G_{3} ;$ D. $\Delta G_{4}$. 


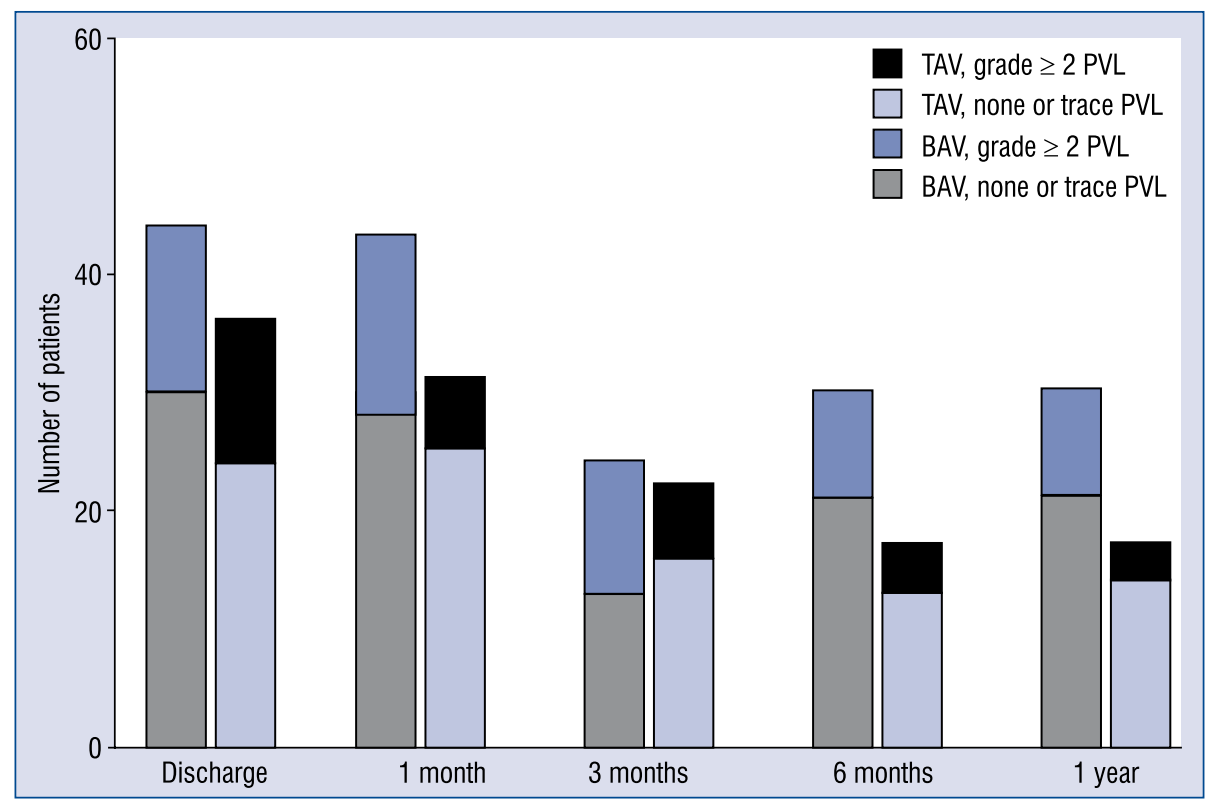

Figure 3. Evolution of paravalvular leak (PVL); BAV — bicuspid aortic valve; TAV — tricuspid aortic valve.

MSCT. Of note, in our reported cohort of patients, $57.65 \%$ were with BAV, which was in line with a previous core lab observation [11]. There could be two main reasons for the high proportion of BAV patients in Chinese TAVI population. The first is genetic variances, which still lacks solid evidence since different proportions of BAV patients exist from center to center [17]. The other explanation is related to patient preference. We see more frequently younger patients of high surgical risk requested TAVI which certainly contributes to this phenomenon. However, no significant difference was found in the proportion of TAV and BAV using $\Delta$ peak velocity on TTE between different time intervals after procedure. This finding, together with similar mid-term post-procedural hemodynamic and clinical outcomes with TAV group in other studies [18, 19], indicates the presence of asymmetric bulky calcification, larger annulus size and possible raphes in BAV patients might not impair THV function. Of note, the number of available TTE follow-ups was small and decreased over time. This was mainly due to the absence of certain follow-up intervals and the fact that some patients did not reach longer follow-up at the time of data retrieval. It is noteworthy that in this study, the self-expanding valve was the only device available at that time. It has been shown in several studies that self-expanding CoreValve prosthesis is associated with a lower residual gradient and greater valve area than balloon-expandable Edwards
SAPIEN prosthesis [20]. This superior antegrade hemodynamic performance could be explained by it being at supra-annular position, thus, it may exert a lower resistance towards left ventricular outflow [20]. Moreover, the longer inflow tract may help impose a lower flow contraction to the valve anatomic orifice. These factors altogether may have contributed to our results.

Another major concern for BAV patients was the risk of PVL, especially with the first-generation THV device. Moderate-to-severe PVL after TAVI has been found to impair outcomes [21, 22]. The presence of raphes, calcifications and difficulties in accurate positioning of the THV contribute to the risk of PVL in BAV patients. Although the incidence of PVL differed across studies with BAV patients, the occurrence of PVL grade $\geq 2$ was generally higher than TAV patients [8, 23, 24]. Similar results were found in our reported cohort. Even though no significant differences were observed in the distribution of PVL severity between BAV and TAV patients, it is possible that the lack of echocardiography data due to incomplete follow-up, which could have affected results. Thus, the high incidence of PVL in BAV patients is still concerning and requires longer follow-up to detect its clinical implications. However, advances in devices allow us to address this complication in BAV patients. Perlman et al. [25] recently reported no more than mild PVL in a cohort of BAV patients treated with the SAPIEN 3 valve at 1 month after the procedure. 
In addition, appropriate computed tomography annular sizing and oversizing strategies are essential in reducing the frequency of PVL or central aortic regurgitation after TAVI [8].

\section{Limitations of the study}

Certain limitations do exist. This is a small, single-center, observational cohort, in which only first-generation self-expanding valves were used. The criteria for at least two serial echocardiographic follow-ups could have decreased the sample size due to incomplete follow-up. Although SV or LVEF were not found to be correlated with gradients or velocities, hemodynamics measured by echocardiography are still easily affected by patient overall functional status at follow-up, which should be taken into consideration when interpreting these results. Moreover, the use of Venus A-valve, which is now the only available type in China, could also limit the value of this study.

\section{Conclusions}

Sustained hemodynamic changes in individual peak velocity and transvalvular gradient differences were observed for 1 year. In this cohort, BAV does not seem to alter hemodynamic changes when compared with its TAV counterparts after TAVI with the self-expanding THV.

\section{Acknowledgements}

We thank Dr. Tsauo Jiay-yu for his help with language revision. This work was supported by the National Hightech Research and Development Program of China (grant number: 2012AA02A510, Beijing, China), the National Natural Science Foundation of China (grant number: 81370219 and 81400267, Beijing, China) and the Supporting Project of Sichuan Provincial Department of Science and Technology (grant numbers: 2012FZ0065 and 2014SZ0004, Sichuan, China).

\section{Conflict of interest: None declared}

\section{References}

1. Douglas PS, Hahn RT, Pibarot P, et al. Hemodynamic outcomes of transcatheter aortic valve replacement and medical management in severe, inoperable aortic stenosis: a longitudinal echocardiographic study of cohort B of the PARTNER trial. J Am Soc Echocardiogr. 2015; 28(2): 210-217.e1, doi: 10.1016/j. echo.2014.10.009, indexed in Pubmed: 25455544.

2. Gotzmann M, Lindstaedt M, Bojara W, et al. Hemodynamic results and changes in myocardial function after transcatheter aortic valve implantation. Am Heart J. 2010; 159(5): 926-932, doi: 10.1016/j.ahj.2010.02.030, indexed in Pubmed: 20435207.

3. Oh JK, Little SH, Abdelmoneim SS, et al. CoreValve U.S. Pivotal Trial Clinical Investigators. Regression of Paravalvular Aortic Regurgitation and Remodeling of Self-Expanding Transcatheter Aortic Valve: An Observation From the CoreValve U.S. Pivotal Trial. JACC Cardiovasc Imaging. 2015; 8(12): 1364-1375, doi: 10.1016/j.jcmg.2015.07.012, indexed in Pubmed: 26508386.

4. Gulino S, Barbanti M, Deste W, et al. Four-year durability of clinical and haemodynamic outcomes of transcatheter aortic valve implantation with the self-expanding CoreValve. EuroIntervention. 2016; 12(8): e1031-e1038, doi: 10.4244/EIJY15M10_08, indexed in Pubmed: 26485733.

5. Barbanti M, Petronio AS, Ettori F, et al. 5-Year Outcomes After Transcatheter Aortic Valve Implantation With CoreValve Prosthesis. JACC Cardiovasc Interv. 2015; 8(8): 1084-1091, doi: 10.1016/j.jcin.2015.03.024, indexed in Pubmed: 26117458.

6. Spethmann S, Dreger H, Baldenhofer G, et al. Long-term Doppler hemodynamics and effective orifice areas of Edwards SAPIEN and medtronic CoreValve prostheses after TAVI. Echocardiography. 2014; 31(3): 302-310, doi: 10.1111/echo.12358, indexed in Pubmed: 24111700.

7. Zegdi R, Ciobotaru V, Noghin M, et al. Is it reasonable to treat all calcified stenotic aortic valves with a valved stent? Results from a human anatomic study in adults. J Am Coll Cardiol. 2008; 51(5): 579-584, doi: 10.1016/j.jacc.2007.10.023, indexed in Pubmed: 18237689.

8. Mylotte D, Lefevre T, Søndergaard L, et al. Transcatheter aortic valve replacement in bicuspid aortic valve disease. J Am Coll Cardiol. 2014; 64(22): 2330-2339, doi: 10.1016/j.jacc.2014.09.039, indexed in Pubmed: 25465419.

9. Nishimura RA, Otto C, Bonow R, et al. 2014 AHA/ACC Guideline for the Management of Patients With Valvular Heart Disease. Journal of the American College of Cardiology. 2014; 63(22): e57-e185, doi: 10.1016/j.jacc.2014.02.536.

10. Cerillo AG, Mariani M, Berti S, et al. Sizing the aortic annulus. Ann Cardiothorac Surg. 2012; 1(2): 245-256, doi: 10.3978/j. issn.2225-319X.2012.06.13, indexed in Pubmed: 23977503.

11. Jilaihawi $\mathrm{H}, \mathrm{Wu} \mathrm{Y}$, Yang $\mathrm{Y}$, et al. Morphological characteristics of severe aortic stenosis in China: imaging corelab observations from the first Chinese transcatheter aortic valve trial. Catheter Cardiovasc Interv. 2015; 85 Suppl 1: 752-761, doi: 10.1002/ ccd.25863, indexed in Pubmed: 25630494.

12. Grube E, Laborde JC, Gerckens U, et al. Percutaneous implantation of the CoreValve self-expanding valve prosthesis in high-risk patients with aortic valve disease: the Siegburg first-in-man study. Circulation. 2006; 114(15): 1616-1624, doi: 10.1161/CIRCULATIONAHA.106.639450, indexed in Pubmed: 17015786.

13. Testa L, Brambilla N, Laudisa ML, et al. Safety and efficacy of the subclavian approach for transcatheter aortic valve implantation with the CoreValve revalving system. Circ Cardiovasc Interv. 2010; 3(4): 359-366, doi: 10.1161/CIRCINTERVENTIONS.109.930453, indexed in Pubmed: 20606135.

14. Zamorano JL, Badano LP, Bruce C, et al. EAE/ASE recommendations for the use of echocardiography in new transcatheter interventions for valvular heart disease. J Am Soc Echocardiogr. 2011; 24(9): 937-965, doi: 10.1016/j.echo.2011.07.003, indexed in Pubmed: 21867869.

15. Kappetein AP, Head SJ, Généreux P, et al. Updated standardized endpoint definitions for transcatheter aortic valve implantation: the Valve Academic Research Consortium-2 consensus docu- 
ment. Eur Heart J. 2012; 33(19): 2403-2418, doi: 10.1093/eurheartj/ehs255, indexed in Pubmed: 23026477.

16. Jilaihawi H, Chen M, Webb J, et al. A Bicuspid Aortic Valve Imaging Classification for the TAVR Era. JACC Cardiovasc Imaging. 2016; 9(10): 1145-1158, doi: 10.1016/j.jcmg.2015.12.022, indexed in Pubmed: 27372022.

17. Cardiovascular Internal Medicine Branch of Chinese Medical Association Professional Committee of Structural Cardiology. Transcatheter aortic valve replacement: consensus from Chinese experts (Chinese). Chin. J. Interv. Cardiol. 2015; 23(12):661-667.

18. Hayashida K, Bouvier E, Lefèvre T, et al. Transcatheter aortic valve implantation for patients with severe bicuspid aortic valve stenosis. Circ Cardiovasc Interv. 2013; 6(3): 284-291, doi: 10.1161/CIRCINTERVENTIONS.112.000084, indexed in Pubmed: 23756698.

19. Kochman J, Huczek Z, Scisło P, et al. Comparison of one- and 12-month outcomes of transcatheter aortic valve replacement in patients with severely stenotic bicuspid versus tricuspid aortic valves (results from a multicenter registry). Am J Cardiol. 2014; 114(5): 757-762, doi: 10.1016/j.amjcard.2014.05.063, indexed in Pubmed: 25037674.

20. Nombela-Franco L, Ruel M, Radhakrishnan S, et al. Comparison of hemodynamic performance of self-expandable CoreValve versus balloon-expandable Edwards SAPIEN aortic valves inserted by catheter for aortic stenosis. Am J Cardiol. 2013; 111(7): 1026-1033, doi: 10.1016/j.amjcard.2012.11.063, indexed in Pubmed: 23351465 .
21. Abdel-Wahab M, Zahn R, Horack M, et al. German transcatheter aortic valve interventions registry investigators. Aortic regurgitation after transcatheter aortic valve implantation: incidence and early outcome. Results from the German transcatheter aortic valve interventions registry. Heart. 2011; 97(11): 899-906, doi: 10.1136/hrt.2010.217158, indexed in Pubmed: 21398694.

22. Leon MB, Smith CR, Mack MJ, et al. PARTNER 2 Investigators, PARTNER Trial Investigators. Two-year outcomes after transcatheter or surgical aortic-valve replacement. N Engl J Med. 2012; 366(18): 1686-1695, doi: 10.1056/NEJMoa1200384, indexed in Pubmed: 22443479.

23. Bauer T, Linke A, Sievert H, et al. Comparison of the effectiveness of transcatheter aortic valve implantation in patients with stenotic bicuspid versus tricuspid aortic valves (from the German TAVI Registry). Am J Cardiol. 2014; 113(3): 518-521, doi: 10.1016/j. amjcard.2013.10.023, indexed in Pubmed: 24342758.

24. Yousef A, Simard T, Webb J, et al. Transcatheter aortic valve implantation in patients with bicuspid aortic valve: A patient level multi-center analysis. Int J Cardiol. 2015; 189: 282-288, doi: 10.1016/j.ijcard.2015.04.066, indexed in Pubmed: 25910593.

25. Perlman GY, Blanke P, Dvir D, et al. Bicuspid Aortic Valve Stenosis: Favorable Early Outcomes With a Next-Generation Transcatheter Heart Valve in a Multicenter Study. JACC Cardiovasc Interv. 2016; 9(8): 817-824, doi: 10.1016/j.jcin.2016.01.002, indexed in Pubmed: 27101906. 